\title{
PREPARATION OF THE WENDELSTEIN 7-X COMMISSIONING
}

\author{
H.-S. Bosch, R. Brakel, M. Gasparotto, D. Hartmann, R. Herrmann, D. Naujoks \\ Max-Planck-Institute for Plasma Physics, D-17491 Greifswald, Germany \\ Bosch@ipp.mpg.de
}

The "fully-optimized" stellarator Wendelstein 7-X is presently under construction at the Max-Planck-Institute for Plasma Physics (IPP), Greifswald, Germany. Assembly of the device, the periphery systems and the diagnostic and heating systems is well advanced and is scheduled to be completed in fall 2014. Recently, a task force has started their work to clarify in detail the commissioning phase, which will commence towards the end of assembly phase.

The commissioning of Wendelstein 7-X consists of two steps with increasing levels of system integration.

I. The local commissioning (LC) of the technical components: This is embedded into the local control system and includes instrumentation and all other peripheral components required. In general, local commissioning will be done before the end of Wendelstein 7-X assembly.

II. The integrated commissioning (IC): This is the step-wise integration of all separate components into the overall system, the central device control and the central data acquisition system.

The following sequence of steps in the integrated commissioning will be followed:

1. Vacuum tests of the cryostat

2. Cryogenic tests of the cryostat

3. Vacuum tests of the plasma vessel

4. Magnet coil systems tests

5. Test of the magnetic field quality

6. In-vessel components tests

7. Preparation for the first plasma

CoDaC (Control, Data acquisition and Communication) plays a crucial role in both commissioning phases: Firstly, local control and the data acquisition of single components is required already for the LC of the respective components. Secondly, full documentation of engineering data and the device control (separated in the central control system and the central safety system) is required, starting from the beginning of the IC phases. These parts will be tested during the IC phases. This paper will discuss the planning of the IC, the risk mitigation, and the schedule in detail. 